\title{
Eta and kaon production in a chiral quark model
}

\author{
B. Golli ${ }^{1 \mathrm{a}}$ and S. Širca ${ }^{2 b}$ \\ 1 Faculty of Education, University of Ljubljana and J. Stefan Institute, 1000 Ljubljana, Slovenia \\ 2 Faculty of Mathematics and Physics, University of Ljubljana and J. Stefan Institute, 1000 Ljubljana, Slovenia
}

June 18, 2021

\begin{abstract}
We apply a coupled-channel formalism incorporating quasi-bound quark-model states to calculate pion scattering into $\eta N, K \Lambda$ and $K \Sigma$ channels, as well $\eta p, \eta n, K^{+} \Lambda$, and $K^{0} \Sigma^{+}$photo-production processes. The meson-baryon and photon-baryon vertices are determined in a SU(3) version of the Cloudy Bag Model. Our model predicts sizable amplitudes in the $P_{11}, P_{13}, P_{33}$ and $S_{11}$ partial waves in agreement with the latest MAID isobar model and the recent partial-wave analyses of the Bonn-Gatchina group. We are able to give a quark-model explanation for the apparent resonance near $1685 \mathrm{MeV}$ in the $\eta n$ channel.
\end{abstract}

\section{Introduction}

The analysis of inelastic scattering and photoproduction of mesons in the framework of quark models which puts strong limits on the meson-baryon and photon-baryon vertices provides important insight into the mechanism of resonance formation and represents a useful guidance to choosing relevant degrees of freedom and parameters in more phenomenological methods used in the partial waveanalyses of large data sets. The channels involved in production of $\eta$ and $K$ mesons give the opportunity to assess the validity of the SU(3) symmetry of the quark model in describing the decay of the resonances. Furthermore, these channels provide invaluable information on the resonances that are weakly coupled to the $\pi N$ channel or overwhelmed by large background and therefore hardly observed in pion production.

We use a coupled-channel formalism incorporating quasi-bound quark-model states to calculate the pion- and photo-production amplitudes in a unified approach. The meson-baryon and photon-baryon vertices are determined in a chiral quark model. We use a $\mathrm{SU}(3)$ extended version of the Cloudy Bag Model (CBM) which includes also the $\rho$ and $\omega$ mesons. The method has been described in detail in our previous papers [1-5] where we have analysed the scattering and electro-production amplitudes in different partial waves. We studied the $\eta N$ channel in the $S_{11}$ partial wave [4], pointing out its important contribution in the region of the $N(1535)$ resonance; we also considered the $K \Lambda$ channel in this partial wave. In the present work we systematically analyse the partial waves with sizable contributions to the $\eta N, K \Lambda$ and $K \Sigma$ decay channels: in addition to $S_{11}$, these are the $P_{11}, P_{13}$ and $P_{33}$ partial waves.

\footnotetext{
a e-mail: bojan.golli@ijs.si

b e-mail: simon.sirca@fmf.uni-lj.si
}

In this work we have decided not to adjust the parameters of the model to fit particular resonances but keep them at the values used in the ground-state calculation; only the positions of the $K$-matrix poles and the mixing angles between the quark model states with the same parity, isospin and angular momentum are considered as adjustable parameters. As we discuss in sec. 2.3, the calculation of different amplitudes proceeds in the same way as in the Cloudy Bag Model. Based on the vast experience gathered in this model we can estimate the reliability of our calculation to (20-30) \%. An agreement on this level with the corresponding partial-wave analysis (PWA) in any given resonance region may be deemed reasonable. However, since no adequate analytic tool has been used to quantitatively verify these estimates, they should be accepted with caution, in particular at higher energies.

Experimentally, photoproduction of $\eta$ mesons has been studied extensively at all modern real-photon facilities. Angular distributions, total cross-sections and several polarization observables have been measured in broad energy ranges; see, for example, Refs. [6-11] for select results on the $\eta p$ channel and Refs. [11-14] for the $\eta n$ channel. The photoproduction of the $\eta$ meson has attracted a particular interest after the GRAAL Collaboration [15] observed a narrow structure at $W \approx 1685 \mathrm{MeV}$ in the $\gamma n \rightarrow \eta n$ reaction which was, however, absent in the $\eta p$ channel. Azimov et al. [16] were the first to discuss the possibility that the structure could belong to a partner of the $\Theta^{+}$ pentaquark in the exotic antidecouplet of baryons. More conventional explanations have attributed the peak to the threshold effect of the $K \Sigma$ channel [17], interference of the nearby $S_{11}, P_{11}$ and $P_{13}$ resonances [18], constructive and destructive interference of the two lowest $S_{11}$ resonances in the $\eta n$ and $\eta p$ channels, respectively, as anticipated in the framework of the Giessen model $[19,20]$ as well as in the Bonn-Gatchina analysis [21,22]. In the framework of the constituent-quark model coupled to the pseudoscalar 
meson octet the (non)appearance of the peak was related to different EM multipoles responsible for excitation in either of the two channels [23]. A similar model applied to $\eta$ production $[24,25]$ has revealed the important role of the lowest $S_{11}, P_{13}, D_{13}$ and the lowest two $F_{15}$ resonances. The photoproduction of $\eta$ mesons off the proton has been extensively studied in a gauge-invariant chiral unitary approach $[26,27]$ with dynamically generated $S 11$ resonances, resulting in a good agreement with data for the $E_{0+}$ pion and $\eta$ amplitudes.

Photo-production of the $K^{+} \Lambda$ final state on the proton has also been a major focus of several recent experimental efforts, resulting in a large body of data on partial and total cross-sections, as well as polarization observables [2836]. Theoretically, pion- and photon-induced $K \Lambda$ production has been studied in different approaches in order to establish the main mechanism governing its behaviour at low energies close to the kaon production threshold. Li et al. $[37,38]$ studied the photoproduction of kaons in a chiral constituent-quark model and stressed the importance of the $S$-wave resonances. Steininger and Meißner [39] and Borasoy et al. [40] studied the threshold behaviour of the kaon photoproduction in the framework of chiral perturbation theory. The Giessen group found sizable contributions from the $S_{11}(1650), P_{13}(1720)$ and $P_{13}(1900)$ resonances [41] and later upon also from the $S_{11}(1535)$, with the background comparable to the resonant terms [42]. The Gent group [43] also concluded that the $S_{11}(1650)$ and $P_{13}(1720)$ resonances contribute most to photo- and electro-production in this channel. Mart [44,45] considered only the $S_{11}(1650)$ resonance and found that the dominant contribution arises from $t$ - and $u$-channel background. The Kent group has included the $\eta N$ and $K \Lambda$ channels in their recent multichannel partial-wave analysis $[46,47]$.

Due to similar energy ranges and particle identification requirements, experimental investigations of the photoproduction of the $K \Sigma$ final state on the proton have gone hand-in-hand with the $K \Lambda$ studies, and we now have a rich data set on both partial and total cross-sections: see, for example, Refs. [28-32] for the $K^{+} \Sigma^{0}$ channel and [48-53] for the $K^{0} \Sigma^{+}$channel. Considering the low-energy photoproduction of the $K \Sigma$ channels close to the threshold, the Giessen group [20] have pointed out the interference of the two low-lying $S_{11}$ resonances, while the $P_{11}$ and $P_{13}$ waves turn out to become important at somewhat higher energies; for the isospin-3/2 channels the $P_{33}$ wave seems to be considerably stronger than the $S_{31}$ wave, in contrast to the previous result [42]. The relatively small cross-section for the $K^{0} \Sigma^{+}$compared to the $K^{+} \Sigma^{0}$ channel is explained by the interference of the resonant and background terms. The Bonn-Gatchina group [54] found similar results regarding the importance of the $S_{11}$ and $P$-wave resonances. Mart [55], on the other hand, considered the $P_{11}(1710), P_{13}(1720), D_{13}(1700)$ and $D_{33}(1700)$ resonances.

A unified description of elastic and inelastic channels including also the production of $\eta$ mesons and kaons has been studied in the dynamical coupled-channel models of the Bonn-Jülich group [56] and the Osaka-Tokyo-Argonne group [57] in order to systematically extract several parameters of the resonances below $2 \mathrm{GeV}$. The Bonn-Jülich results for the pion-induced reactions confirm the dominant role of the $S_{11}$ wave at lower energies in the isospin$1 / 2$ channels, and the $P_{33}$ wave in the isospin-3/2 ones.

In the next section we briefly overview the calculation of the pion- and photo-production amplitudes in our coupled-channel approach for the $K$ matrix and introduce the $\mathrm{SU}(3)$ version of the CBM. We discuss the resonances included in the $P_{13}$ partial wave which has not been considered in our previous work. We also discuss in more detail the $P_{11}$ wave resonances since we have now included several new channels with respect to our previous calculation.

In sect. 3 we present the scattering amplitudes involving $\eta N, K \Lambda$ and $K \Sigma$ channels, and in sect. 4 the photoproduction amplitudes. We discuss in detail the difference between the $\eta$ photoproduction amplitudes on the proton and on the neutron. We give a possible quark-model mechanism for this difference which may explain the apparent resonant structure in the total cross-section near $1685 \mathrm{MeV}$. The results are summarized in sect. 5 .

\section{The model}

\subsection{The coupled channel formalism}

In chiral quark models in which mesons couple linearly to the quark core the elements of the $K$ matrix in the basis with good total angular momentum $J$ and isospin $I$ take the form [2]:

$$
K_{M^{\prime} B^{\prime} M B}^{J I}=-\pi \mathcal{N}_{M^{\prime} B^{\prime}}\left\langle\Psi_{J I}^{M B}\left\|V^{M^{\prime}}(k)\right\| \Psi_{B^{\prime}}\right\rangle,
$$

where $V^{M^{\prime}}(k)$ stands for the quark-meson vertex of the underlying quark model, $\Psi_{B^{\prime}}$ is the baryon state in the $M^{\prime} B^{\prime}$ channel, and $\mathcal{N}_{M B}=\sqrt{\omega_{M} E_{B} /\left(k_{M} W\right)}$. Here $W$ is the invariant energy, $E_{B}$ is the baryon energy and $\omega_{M}$ and $k_{M}$ are the meson energy and momentum. The expression (1) differs from the expression for the corresponding $T$ matrix in that the incoming state is replaced by the principal-value state $\Psi_{J I}^{M B}$; in the following, all integrals are assumed in the principal value sense. The quark-model quasi-bound states $\Phi_{\mathcal{R}}$ are included through the following ansatz:

$$
\begin{aligned}
\left|\Psi_{J I}^{M B}\right\rangle= & \mathcal{N}_{M B}\left\{\left[a^{\dagger}\left(k_{M}\right)\left|\Psi_{B}\right\rangle\right]^{J I}+\sum_{\mathcal{R}} c_{\mathcal{R}}^{M B}\left|\Phi_{\mathcal{R}}\right\rangle\right. \\
& \left.+\sum_{M^{\prime} B^{\prime}} \int \frac{\mathrm{d} k \chi^{M^{\prime} B^{\prime} M B}\left(k, k_{M}\right)}{\omega_{k}+E_{B^{\prime}}(k)-W}\left[a^{\dagger}(k)\left|\Psi_{B^{\prime}}\right\rangle\right]^{J I}\right\},
\end{aligned}
$$

where the first term represents the free meson $(\pi, \eta, K$, $\ldots)$ and the baryon $(N, \Delta, \Lambda, \ldots)$ and defines the channel, the next term is the sum over bare three-quark states, 
while the third term describes meson clouds around different isobars. The principal-value states (2) are normalized as

$$
\left\langle\Psi^{\alpha}(W) \mid \Psi^{\beta}\left(W^{\prime}\right)\right\rangle=\delta\left(W-W^{\prime}\right)\left[\delta_{\alpha, \beta}+\mathbf{K}_{\alpha, \beta}^{2}\right] .
$$

They are not orthonormal; the orthonormalized states are constructed by inverting the norm.

The meson amplitudes $\chi^{M^{\prime} B^{\prime} M B}\left(k, k_{M}\right)$ and the coefficients $c_{\mathcal{R}}^{M B}$ are obtained from the Kohn variational principle leading to a set of coupled equations: equations of the Lippmann-Schwinger type for the meson amplitudes

$$
\begin{aligned}
& \chi^{M^{\prime} B^{\prime} M B}\left(k, k_{M}\right)=-\sum_{\mathcal{R}} c_{\mathcal{R}}^{M B} V_{B^{\prime} \mathcal{R}}^{M^{\prime}}(k) \\
& +\mathcal{K}^{M^{\prime} B^{\prime} M B}\left(k, k_{M}\right) \\
& +\sum_{M^{\prime \prime} B^{\prime \prime}} \int \mathrm{d} k^{\prime} \frac{\mathcal{K}^{M^{\prime} B^{\prime} M^{\prime \prime} B^{\prime \prime}}\left(k, k^{\prime}\right) \chi^{M^{\prime \prime} B^{\prime \prime} M B}\left(k^{\prime}, k_{M}\right)}{\omega_{k}^{\prime}+E_{B^{\prime \prime}}\left(k^{\prime}\right)-W},
\end{aligned}
$$

and equations for the coefficients $c_{\mathcal{R}}^{M B}$ :

$$
\begin{aligned}
\left(W-M_{\mathcal{R}}^{(0)}\right) c_{\mathcal{R}}^{M B} & =V_{B \mathcal{R}}^{M}\left(k_{M}\right) \\
& +\sum_{M^{\prime} B^{\prime}} \int \mathrm{d} k \frac{\chi^{M^{\prime} B^{\prime} M B}\left(k, k_{M}\right) V_{B^{\prime} \mathcal{R}}^{M^{\prime}}(k)}{\omega_{k}+E_{B^{\prime}}(k)-W} .
\end{aligned}
$$

Here

$$
\begin{aligned}
\mathcal{K}^{M^{\prime} B^{\prime}} M B & \left(k, k^{\prime}\right)=\sum_{B^{\prime \prime}} f_{B B^{\prime}}^{B^{\prime \prime}} \frac{\mathcal{V}_{B^{\prime \prime} B^{\prime}}^{M^{\prime}}\left(k^{\prime}\right) \mathcal{V}_{B^{\prime \prime} B}^{M}(k)}{\omega_{k}+\omega_{k}^{\prime}+E_{B^{\prime \prime}}(\bar{k})-W}, \\
f_{A B}^{C} & =\sqrt{\left(2 J_{A}+1\right)\left(2 J_{B}+1\right)\left(2 I_{A}+1\right)\left(2 I_{B}+1\right)} \\
& \times W\left(l_{B} J_{A} J_{B} l_{A} ; J_{C}, J\right) W\left(i_{B} I_{A} I_{B} i_{A} ; I_{C}, I\right)
\end{aligned}
$$

$l_{A}, i_{A}, \ldots$ are the meson, $J_{A}, I_{A}, \ldots$ are the baryon angular momenta and isospins, $V_{B \mathcal{R}}^{M}(k)$ are the bare matrix elements of the quark-meson interaction between the baryon state $B$ and the bare three-quark state $\Phi_{\mathcal{R}}$, and $M_{\mathcal{R}}^{(0)}$ is the energy of the bare state. The corresponding dressed vertices obey the equation

$$
\mathcal{V}_{B \mathcal{R}}^{M}=V_{B \mathcal{R}}^{M}(k)+\sum_{M^{\prime} B^{\prime}} \int \mathrm{d} k^{\prime} \frac{\mathcal{K}^{M B M^{\prime} B^{\prime}}\left(k, k^{\prime}\right) \mathcal{V}_{B^{\prime} \mathcal{R}}^{M^{\prime}}\left(k^{\prime}\right)}{\omega_{k}^{\prime}+E_{B^{\prime}}\left(k^{\prime}\right)-W}
$$

Solving the coupled set of equations can be considerably simplified by using a separable approximation for the kernel of the form

$$
\begin{aligned}
\frac{1}{\omega_{k}+\omega_{k}^{\prime}+E_{B^{\prime \prime}}-W} \approx \\
\frac{\left(\omega_{M}+\omega_{M^{\prime}}+E_{B^{\prime \prime}}-W\right)}{\left(\omega_{k}+E_{B^{\prime \prime}}-E_{B^{\prime}}\right)\left(\omega_{k}^{\prime}+E_{B^{\prime \prime}}-E_{B}\right)}
\end{aligned}
$$

where $W=E_{B}+\omega_{M}=E_{B^{\prime}}+\omega_{M^{\prime}}$. The approximation on the RHS of (8) coincides with the exact expression on the
LHS when either of the two pions is on-shell. Furthermore, the dressed vertices in (6) can be replaced by the bare ones in those cases in which the mesons are only weakly coupled to baryons. It turns out that in the case of the P33 and P11 partial wave, in which the $p$-wave pions strongly couple to the quark core, solving the integral equations alters the coupling constants by some $50 \%$ with respect to the bare quark values; in the case of $s$ - and $d$-wave pions as well as other mesons, the modification typically remains at the level of $5 \%$ to $10 \%$. Let us note that neglecting the integrals in (4), (5) and (7) corresponds to the so-called Born approximation for the $K$ matrix.

The meson amplitudes are proportional to the half offshell matrix elements of the $K$-matrix,

$$
K_{M^{\prime} B^{\prime} M B}\left(k, k_{M}\right)=\pi \mathcal{N}_{M^{\prime} B^{\prime}} \mathcal{N}_{M B} \chi^{M^{\prime} B^{\prime} M B}\left(k, k_{M}\right)
$$

The procedure outlined above yields the final expression

$$
\begin{aligned}
K_{M^{\prime} B^{\prime} M B}\left(k, k_{M}\right)= & -\sum_{\mathcal{R}} \frac{\mathcal{V}_{B \mathcal{R}}^{M}\left(k_{M}\right) \mathcal{V}_{B^{\prime} \mathcal{R}}^{M^{\prime}}(k)}{Z_{\mathcal{R}}(W)\left(W-W_{\mathcal{R}}\right)} \\
& +K_{M^{\prime} B^{\prime} M B}^{\mathrm{bkg}}\left(k, k_{M}\right),
\end{aligned}
$$

where the first term represents the contribution of various resonances, while the second term originates in the nonresonant background processes; $Z_{\mathcal{R}}$ is the resonance wave function normalization.

The scattering $T$ matrix is obtained by solving the Heitler equation $T=K+\mathrm{i} K T$.

\subsection{The underlying quark model}

The vertices are calculated in a version of the Cloudy Bag Model extended to the pseudo-scalar, $\phi_{a}$, and vector, $\boldsymbol{A}_{a}$, $\mathrm{SU}(3)$ meson octet [58]:

$$
\begin{aligned}
H_{\mathrm{int}}= & -\int \mathrm{d} \boldsymbol{r}\left[\frac{\mathrm{i}}{2 f} \bar{q} \lambda_{a}\left(\gamma_{5} \phi_{a}+\gamma \cdot \boldsymbol{A}_{a}\right) q \delta_{S}\right. \\
& \left.+\frac{1}{4 f^{2}} \bar{q} \lambda_{a} \gamma^{\mu} q\left(\phi \times \partial_{\mu} \phi\right)_{a} \theta_{V}\right], \\
& a=1,2, \ldots, 8 .
\end{aligned}
$$

The model provides a consistent parametrization of the meson-baryon and photon-baryon coupling constants and form factors in terms of $f$ (equivalent to $f_{\pi}$ ) and the bag radius $R_{\text {bag }}$. We use $R_{\text {bag }}=0.83 \mathrm{fm}$ and $f=76 \mathrm{MeV}$, consistent with the values used in the ground-state calculations. In addition, the bare masses of the resonances are also free parameters. The last term in (11) has not been included in our previous calculations. It contributes to the background and influences most strongly the real part of the elastic $\pi N$ amplitude in the $P_{13}$ and $P_{31}$ partial waves. Using the same value for $f$ as in the first term would result in a too strong contribution of this term. We therefore multiply this term by a factor $\frac{1}{2}$. Furthermore, the strength of $\rho$ meson coupling should be reduced by a factor of 3 , consistent with the value of the $\rho$ meson decay constant $f_{\rho} \approx 200 \mathrm{MeV}$. 


\subsection{The photo- and electroproduction amplitudes}

In the case of photo- and electroproduction the strong vertex in (1) is replaced by the electromagnetic interaction vertex. In chiral quarks models with the underlying Lagrangian, such as the Cloudy Bag Model used in our calculation, the electromagnetic currents are derived using the principle of minimal coupling. The quark, pion and kaon contributions to the spatial part of the electromagnetic current read

$$
\begin{aligned}
\boldsymbol{j}_{E M}^{q} & =\sum_{i=1}^{3} \bar{\psi} \gamma(i) \psi\left(\frac{1}{6}+\frac{\tau_{0}(i)}{2}\right), \\
\boldsymbol{j}_{E M}^{\pi} & =\mathrm{i} \sum_{t} t \pi_{t}(\boldsymbol{r}) \boldsymbol{\nabla} \pi_{-t}(\boldsymbol{r}), \\
\boldsymbol{j}_{E M}^{K} & =\mathrm{i}\left[K^{-}(\boldsymbol{r}) \boldsymbol{\nabla} K^{+}(\boldsymbol{r})-K^{+}(\boldsymbol{r}) \boldsymbol{\nabla} K^{-}(\boldsymbol{r})\right] .
\end{aligned}
$$

The resonant part of the electroproduction amplitude can be cast in the form

$$
\mathcal{M}_{M B \gamma N}^{\mathrm{res}}=\sqrt{\frac{\omega_{\gamma} E_{N}^{\gamma}}{\omega_{\pi} E_{N}}} \frac{\xi}{\pi \mathcal{V}_{N \mathcal{R}}^{\pi}}\left\langle\widehat{\Psi}_{\mathcal{R}}\left|V_{\gamma}\right| \Psi_{N}\right\rangle T_{M B \pi N}
$$

where $V_{\gamma}$ describes the interaction of the photon with the electromagnetic current and $\xi$ is the spin-isospin factor depending on the considered multipole and the spin and isospin of the outgoing hadrons. The resonance state $\widehat{\Psi}_{\mathcal{R}}$ is extracted from the components in the second and the third term in (2) that are proportional to the resonance pole $\left(W-W_{\mathcal{R}}\right)^{-1}$; it involves the bare-quark core and the meson cloud:

$\left|\widehat{\Psi}_{\mathcal{R}}\right\rangle=Z_{\mathcal{R}}^{-\frac{1}{2}}\left[\left|\Phi_{\mathcal{R}}\right\rangle-\sum_{M B} \int \frac{\mathrm{d} k \mathcal{V}_{B \mathcal{R}}^{M}(k)}{\omega_{k}+E_{B}-W}\left[a^{\dagger}(k)\left|\Psi_{B}\right\rangle\right]^{J I}\right]$.

Let us note that the calculation of the electromagnetic vertices in our approach proceeds in the same way as in the standard calculation in the framework of the Cloudy Bag Model.

Although the corresponding quark-level Lagrangian of the Cloudy Bag Model respects electromagnetic gauge invariance, this may not be the case on the nucleon level. Miller and Thomas [59] have shown that the model respects gauge invariance in the Breit frame. In this frame, no extra terms are needed to calculate the nucleon electromagnetic form factors. The fact that their result applies only to a specific frame should be a caveat to the reader, since this invariance should hold in all frames. In the calculation of form-factors no attempt is usually made to construct states with good linear momentum. In Ref. [60] the amplitudes for electroproduction of the $\Delta$ resonance have been calculated using the Peierls-Thouless method to eliminate the spurious center of mass motion. This has altered the results obtained in the static approximation by about $5 \%$ to $8 \%$ and enabled the authors to estimate the error of neglecting recoil effects to $q^{2} / 4 m_{N}^{2}, q^{2}$ being the pion momentum squared. Accordingly, we expect these deficiencies to map to our computed amplitudes at a comparable level of uncertainty, not exceeding $20 \%$ below $q^{2} \approx 1 \mathrm{GeV}^{2}$. A considerably larger disagreement might indicate a more severe inconsistency in the quarkmodel picture of a specific resonance. Another source of discrepancy could be a strong contribution of the background processes, in particular at higher energies around $\sim 1700 \mathrm{MeV}$ and above, not included or strongly underestimated in our calculation.

\subsection{Resonances in the $P_{13}$ partial wave}

In the quark model, the $N(1720) \frac{3}{2}^{+}$and $N(1900) \frac{3}{2}^{+}$resonances are obtained by exciting one $s$ quark to the $d$ orbit with either $j=\frac{3}{2}$ or $j=\frac{5}{2}$. (Note that the $p$-wave pseudoscalar mesons couple only to $j=\frac{3}{2}$.)

The two physical resonances are linear combinations of the spin doublet and spin quadruplet states:

$$
\begin{aligned}
& |N(1720)\rangle=\cos \vartheta_{p}\left|\mathbf{7 0},{ }^{2} \mathbf{8}, J=\frac{3}{2}\right\rangle-\sin \vartheta_{p}\left|\mathbf{7 0},{ }^{4} \mathbf{8}, J=\frac{3}{2}\right\rangle, \\
& |N(1900)\rangle=\sin \vartheta_{p}\left|\mathbf{7 0},{ }^{2} \mathbf{8}, J=\frac{3}{2}\right\rangle+\cos \vartheta_{p}\left|\mathbf{7 0},{ }^{4} \mathbf{8}, J=\frac{3}{2}\right\rangle .
\end{aligned}
$$

Here $\mathbf{7 0}$ stands for the SU(6) flavour-spin multiplet and 8 corresponds to isospin $I=\frac{1}{2}$. The detailed structure in terms of the $j j$-coupled states is given in Appendix A.

The mixing originates from the gluon-quark and the meson-quark interaction. The mixing due to gluon interaction has been investigated in the constituent-quark model [61] and in the bag model [62,63]. In our model, the mixing arises also from meson-baryon interaction as described in [2]. The gluon interaction is not included in our model and the corresponding mixing angle is taken as a free parameter. The meson-loop contribution to the mixing angle is $W$-dependent. In this partial wave the angle starts from $-15^{\circ}$, reaches $-30^{\circ}$ in the region of the lower resonance, and returns to $-15^{\circ}$ in the region of the upper resonance. The $T$ matrix for the elastic channel is shown in fig. 1 in a coupled-channel calculation including $\pi N, \pi \Delta, \sigma_{l=2} N, \eta N, \pi N(1440), \rho_{l=0} N, K \Lambda, \rho_{l=2} N, K \Sigma$, $\omega_{l=0} N$ and $\omega_{l=2} N$ channels.

\subsection{The Roper resonances revisited}

The $N(1440) \frac{1}{2}^{+}$resonance was extensively studied in our previous work $[2,3,67]$, assuming the $\pi \Delta$ and $\sigma N$ inelastic channels. In order to reproduce the behaviour of the scattering amplitudes up to $\approx 1800 \mathrm{MeV}$, we included also the $N(1710) \frac{1}{2}^{+}$resonance assuming it decayed only into the $\sigma N$ channel. If we want to study the production of strange mesons in the $P_{11}$ partial wave, we have to specify its quark configuration, which is not obvious; it may consist of the $(1 s)^{3},(1 s)^{2}(2 s)^{1},(1 s)^{1}(2 s)^{2},(1 s)^{1}(1 p)^{2},(1 s)^{1}(1 d)^{2} \ldots$ configurations or even a component with the excitation of the $\sigma$ cloud [67]. If we limit ourselves to the type of excitation in which only a single $1 s$ quark is raised to a higher orbit, it suffices to specify only the admixture of $(1 s)^{2}(2 s)^{1}$ (and eventually of $\left.(1 s)^{2}(3 s)^{1}\right)$ configuration in 


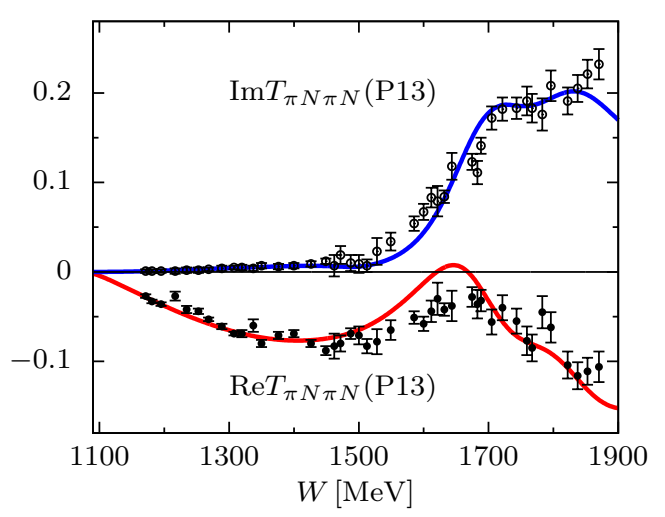

Fig. 1. The real and the imaginary part of the scattering $T$ matrix for the $P_{13}$ partial wave. The data points are from the SAID $\pi N \rightarrow \pi N$ partial-wave analysis $[65,66]$.

the resonance state. We therefore assume the following structure of the two Roper resonances:

$$
\begin{aligned}
& N(1440)=\cos \vartheta_{R}(1 s)^{2}(2 s)^{1}-\sin \vartheta_{R}(1 s)^{1}(1 x)^{2}, \\
& N(1710)=\sin \vartheta_{R}(1 s)^{2}(2 s)^{1}+\cos \vartheta_{R}(1 s)^{1}(1 x)^{2},
\end{aligned}
$$

where $x$ stands for $l>0$ quark orbits not involved in the transition matrix elements.

The $N(1440) \frac{1}{2}^{+}$and the nucleon also mix, which leads to the important nucleon-pole contribution to various amplitudes. This mixing is calculated through the quark interaction with the meson cloud (meson loops) as discussed in [2] and does not introduce additional adjustable parameters.

The $\Delta(1232) \frac{3}{2}^{+}$and the $\Delta(1600) \frac{3}{2}^{+}$resonances are linear superposition of the $(1 s)^{3}$ and $(1 s)^{2}(2 s)^{1}$ spin- $3 / 2$ configurations with the mixing angle calculated through the pion loops.

\subsection{Resonances in the $S_{11}$ partial wave}

The negative-parity resonances $N(1535) \frac{1}{2}^{-}$and $N(1650) \frac{1}{2}$ have been extensively studied in our previous work [4]. Here the meson loops are less important compared to the $P$-wave resonances and the mixing arises primarily through the gluons [61-63]:

$$
\begin{aligned}
& |N(1535)\rangle=\cos \vartheta_{s}\left|\mathbf{7 0},{ }^{2} \mathbf{8}, J=\frac{1}{2}\right\rangle-\sin \vartheta_{s}\left|\mathbf{7 0},{ }^{4} \mathbf{8}, J=\frac{1}{2}\right\rangle \\
& |N(1650)\rangle=\sin \vartheta_{s}\left|\mathbf{7 0},{ }^{2} \mathbf{8}, J=\frac{1}{2}\right\rangle+\cos \vartheta_{s}\left|\mathbf{7 0},{ }^{4} \mathbf{8}, J=\frac{1}{2}\right\rangle .
\end{aligned}
$$

The structure of these resonances in terms of the $j j$-coupled states involving quark excitation to $p_{1 / 2}$ and $p_{3 / 2}$ orbits is given in [4]. In our previous work we made some adjustment of the quark-pion coupling strength in order to better reproduce the imaginary part of the amplitude in the elastic channel and in the $d$-wave $\pi \Delta$ channel. In the present approach we keep the constants at their quarkmodel values. The mixing angle that best reproduces the $\eta N$ to $\pi N$ branching ratio is close to $-30^{\circ}$ and is only weakly affected by the meson loops.

The isospin-3/2 partner $\Delta(1650)=\left|\mathbf{7 0},{ }^{2} \mathbf{1 0}, J=\frac{1}{2}\right\rangle$ contributes very weakly to the amplitudes considered in this work and will not be included in further analysis. The same holds for the contribution of the $D$-wave resonances treated in our previous work [5].

\section{Scattering amplitudes involving $\eta N, K \Lambda$ and $K \Sigma$ channels}

Figure 2 shows the scattering amplitudes for the $\pi N \rightarrow$ $\eta N, \pi N \rightarrow K \Lambda$ and $\pi N \rightarrow K \Sigma$ reactions in the $S_{11}, P_{11}$ and $P_{13}$ partial waves.

For the $\pi N \rightarrow \eta N$ channel the dominant $S_{11}$ wave is very well reproduced compared to the Bonn-Gatchina analysis close to the threshold and in the region of the lower resonance; in the region of the upper resonance, the amplitudes follow the general trend and drop almost to zero. In the $P_{13}$ partial wave our model predicts a too strong $\eta N$ amplitude, almost insensitive to the mixing angle. If we use a different value of the coupling constant, consistent with the decay constant $f_{\eta} \approx 1.2 f_{\pi}$, the amplitude would drop by $20 \%$ but would still be too large compared to values from different partial-wave analyses. The situation in the $P_{11}$ partial wave is rather intriguing because of many distinct processes contributing to the amplitude: the nucleon pole, the two Roper resonances and the $u$-channel process. Our results in fig. 2 are nonetheless not inconsistent with various partial-wave analyses.

The agreement of the calculated $\pi N \rightarrow K \Lambda$ amplitudes with the partial-wave analyses is good and would further improve if we modified the quark-kaon coupling constant according to $f_{K}=1.2 f_{\pi}$. While the $\eta N$ amplitude overshoots the PWA data in the $P_{13}$ partial wave, in the $K \Lambda$ case this happens in the $P_{11}$ wave.

The strength of the amplitudes in the $K \Sigma$ channel compares well with still rather uncertain analysis of the Bonn-Gatchina group, even without adjustment of the coupling constant (see fig. 2, right column).

In fig. 3 the results for the $P_{33}$ partial wave are shown. Also in this partial wave the magnitude of the scattering amplitudes agrees well with the Bonn-Gatchina analysis.

\section{Photoproduction}

Photoproduction involves the calculation of EM vertices in the framework of the same underlying quark model. In general the photoexcitation amplitudes calculated in the CBM do not compare so favourably with experiment as in the case of strong vertices; already the magnetic moments of the nucleon are considerably underestimated if we insist on our value of the bag radius. This should be kept in mind when assessing the quality of our results.

In our previous work we calculated the electric multipoles from the charge density using the current conservation, since - in particular for the $E_{1+}$ multipole for 

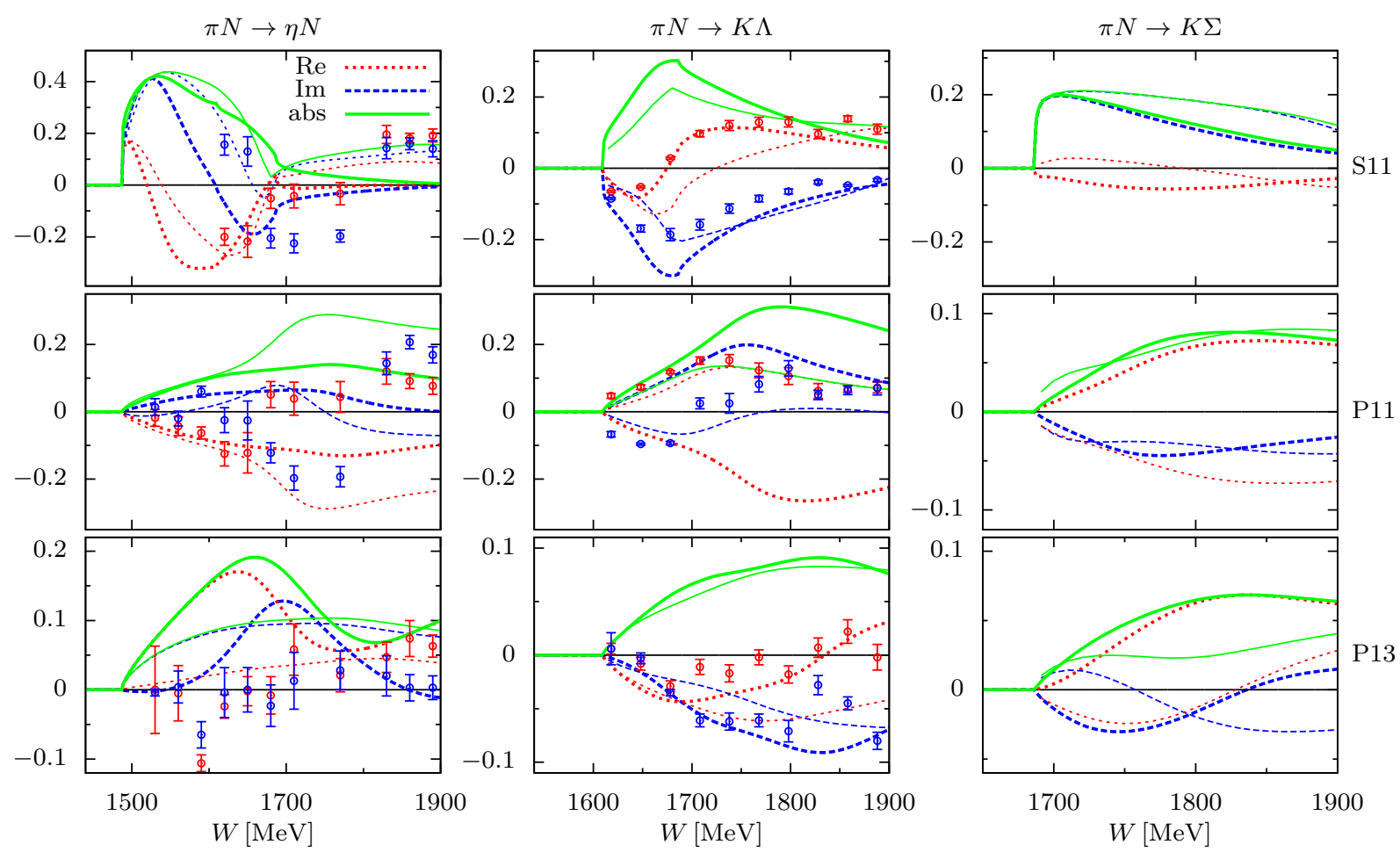

Fig. 2. (Color online.) The real and imaginary parts and the absolute values of the scattering $T$ matrix for the $\eta N, K \Lambda$ and $K \Sigma$ channels in the $S_{11}, P_{11}$ and $P_{13}$ partial waves (from top to bottom row). The corresponding thin curves denote the 2014-2 solution of the Bonn-Gatchina group [68]. The data points are from the single-energy analyses of Ref. [70].

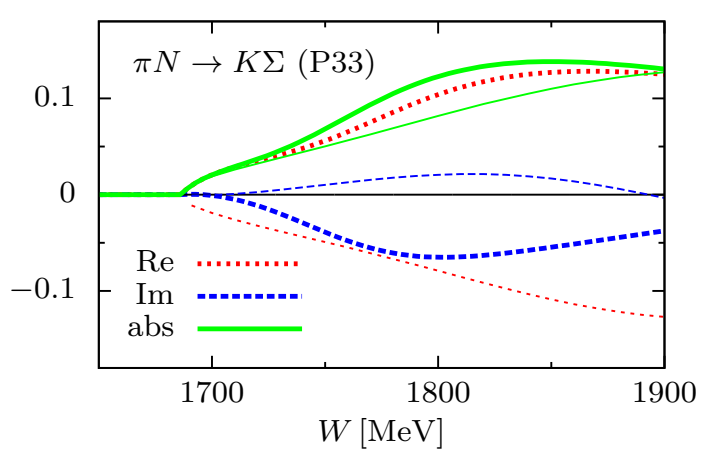

Fig. 3. (Color online.) The real and imaginary part of the scattering $T$ matrix for the $K \Sigma$ channel in the $P_{33}$ partial wave. Notation as in fig. 2.

the $\Delta(1232)$ excitation - the results were less sensitive to the small variations of the model parameters [69]. In the present work we strictly calculate both, the magnetic as well as the electric multipoles, from the currents (12)(14).

\section{1 $\eta p$ and $\eta n$ photoproduction}

Photoproduction of $\eta$ on the nucleon is particularly interesting because of the presence of an apparent narrow resonance very close to the $K \Sigma$ threshold. The intriguing feature of this structure is that it appears only in the $\gamma n \rightarrow \eta n$ channel and is completely absent in the $\gamma p \rightarrow \eta p$ channel.

The photoproduction amplitudes are dominated by the $E_{0+}$ multipole and are reasonably well reproduced in our model (see fig. 4); the neutron amplitude turns out to be somewhat weaker in the region of the lower resonance, while the imaginary parts of both amplitudes are underestimated in the region of the upper resonance. ${ }^{1}$ The sign of our amplitudes agrees with those of the MAID analysis but is opposite to that of the Bonn-Gatchina group - to facilitate the comparison we have reversed the sign of the latter in fig. 4.

The $M_{1-}, M_{1+}, E_{1+}$ are less precisely determined in the partial-wave analyses of the MAID and Bonn-Gatchina groups; our results in fig. 5 reproduce the correct order of magnitude and overestimate the $E_{1}+$ amplitude, in accordance with a too large strong amplitude in this partial wave.

The total cross-section is displayed in fig. 6 and compared to the results obtained from the Bonn-Gatchina 2014-2 analysis (which fits with great precision the experimental cross-section). The dominant $E_{0+}$ contribution is shown separately. Our results do show a structure in the region of the $K \Sigma$ threshold in the $\gamma n$ channel, which is

\footnotetext{
${ }^{1}$ In our previous work (see [4]) there was an error in the calculation of the neutron amplitudes, namely the contribution involving the excitation of the $p_{3 / 2}$ orbit was taken with the wrong sign.
} 

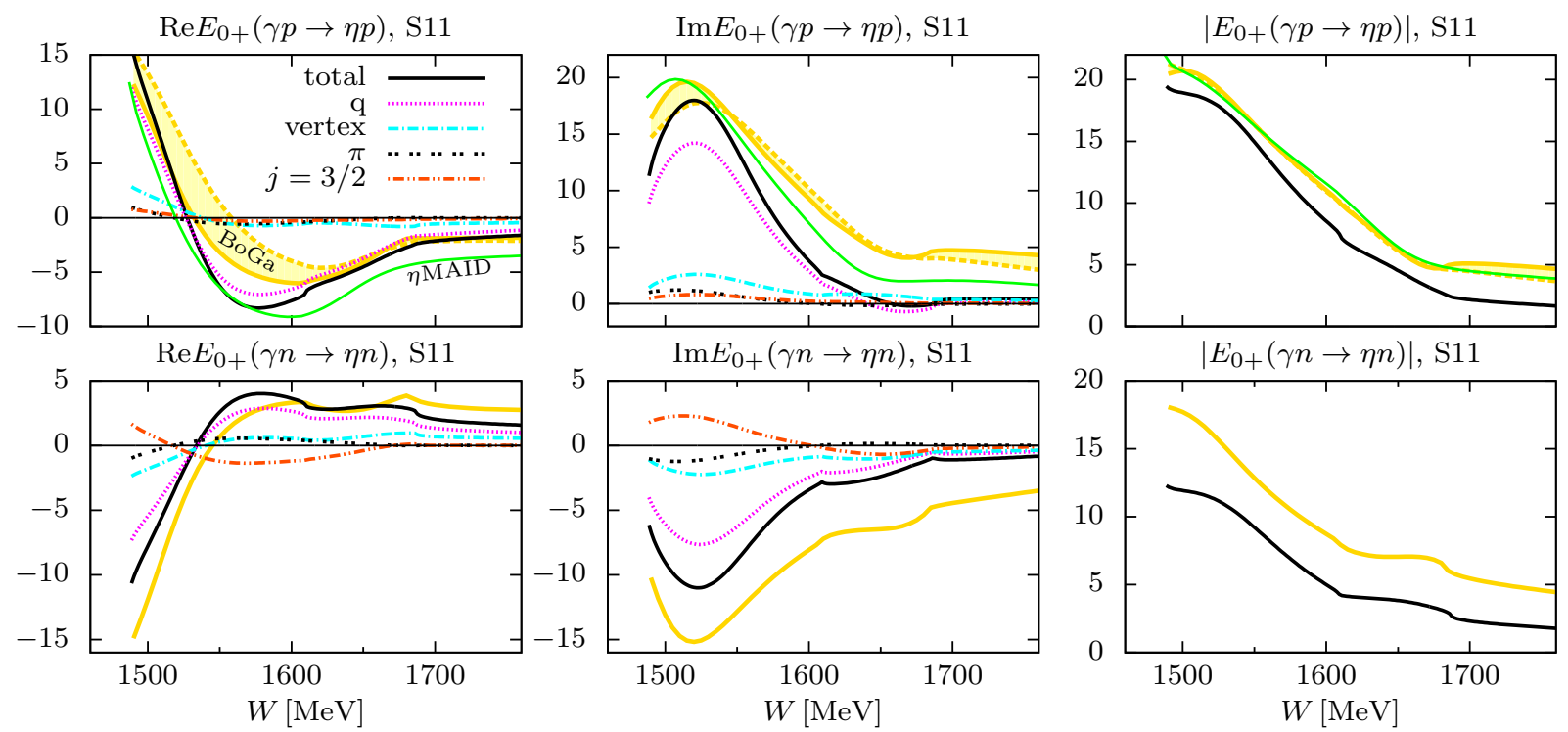

Fig. 4. (Color online.) The real and imaginary parts and the absolute values of the $E_{0+}$ multipole in $\eta$ photoproduction (in units of $\mathrm{mfm}$ ). The Bonn-Gatchina solution (yellow) is multiplied by -1 ; for $p \gamma$ the dashed lines correspond to the 2014-1 and the solid lines to the 2014-2 solution. For better visibility, the two Bonn-Gatchina solutions (unless only one of them is available) are connected by shading. The $\eta$ MAID results (green) for $\gamma p$ are from [71]. The quark, vertex-correction and pion-cloud contributions are shown and, separately, the contribution from the $p_{3 / 2}$ orbit.
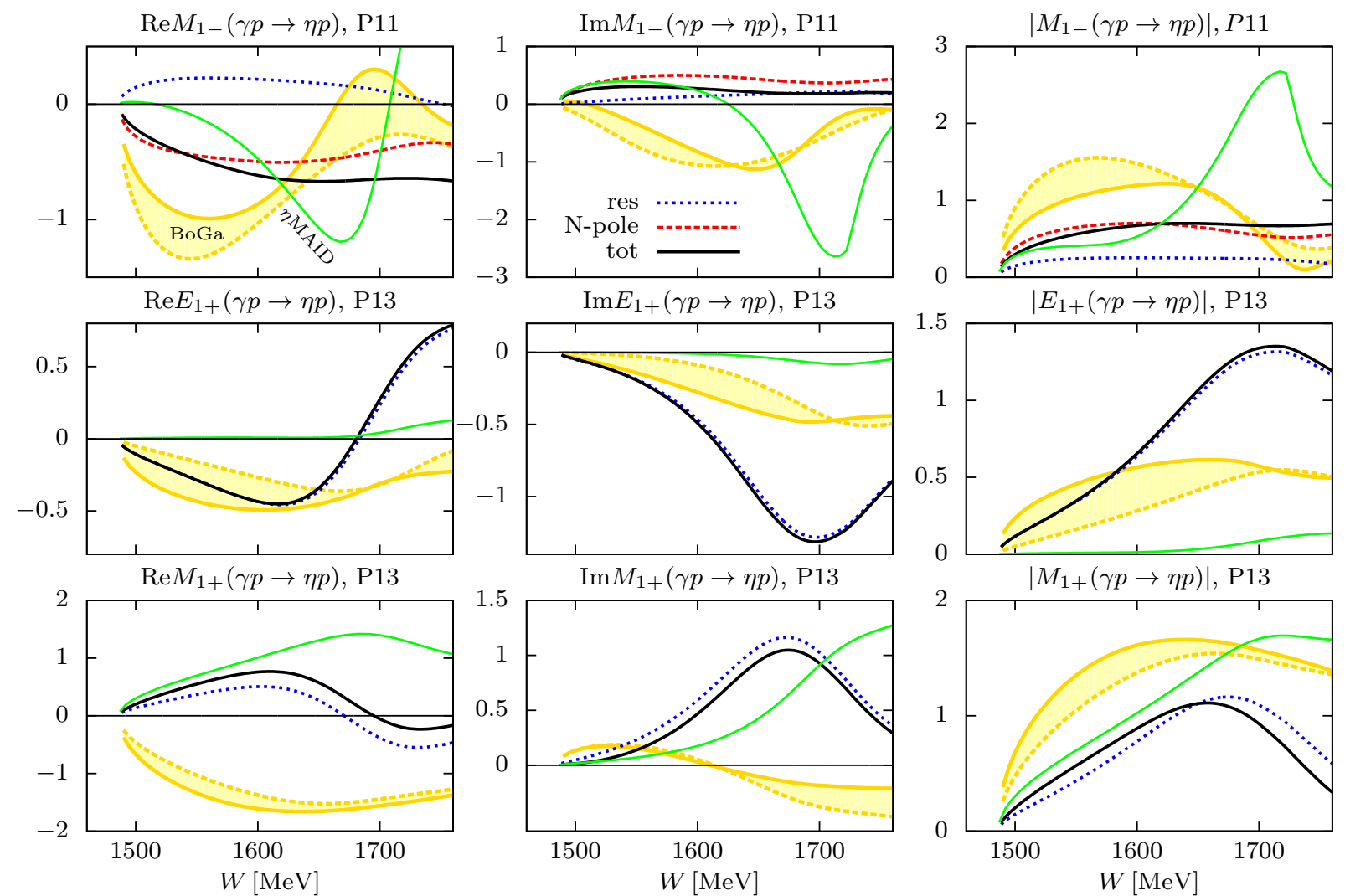

Fig. 5. (Color online.) The real and imaginary parts and the absolute values of the $M_{1-}, E_{1+}$ and $M_{1+}$ multipoles in $\eta$ photoproduction. Notation as in fig. 4 ; the resonance (dots) and the nucleon-pole (dashes, in the $P_{11}$ wave) contributions are also shown. 
absent in the $\gamma p$ channel, though its strength is lower compared to the experiment. ${ }^{2}$ Nonetheless, our analysis offers a possible and straightforward explanation of this structure in terms of the quark model: a combination of a peculiar property of the (relativistic) wave functions of the $S_{11}$ resonances and the presence of the $K \Sigma$ threshold. Let us start from the states in (19) expressed in terms of the quark $p_{j}$ orbits [63]:

$$
\begin{aligned}
\left|{ }^{4} \boldsymbol{8}_{\frac{1}{2}}\right\rangle= & \frac{1}{3}\left|(1 s)^{2}\left(1 p_{3 / 2}\right)^{1}\right\rangle+\frac{\sqrt{8}}{3}\left|(1 s)^{2}\left(1 p_{1 / 2}\right)^{1}\right\rangle \\
\left|{ }^{2} \boldsymbol{8}_{\frac{1}{2}}\right\rangle= & -\frac{2}{3}\left|(1 s)^{2}\left(1 p_{3 / 2}\right)^{1}\right\rangle+\frac{\sqrt{2}}{6}\left|(1 s)^{2}\left(1 p_{1 / 2}\right)^{1}\right\rangle \\
& +\frac{\sqrt{2}}{2}\left|(1 s)^{2}\left(1 p_{1 / 2}\right)^{1}\right\rangle^{\prime}
\end{aligned}
$$

where the last two components with $p_{1 / 2}$ correspond to coupling the two $s$-quarks to spin 1 and 0 , respectively; the flavour (isospin) part is not written explicitly. In the strong part of the production amplitude, the $s$-wave $\eta$ meson couples only to the $p_{1 / 2}$ quark, while the electric dipole $(E 1)$ photon couples to the $p_{1 / 2}$ component as well as to the $p_{3 / 2}$ component. For the proton, however, the isoscalar part of the charge operator exactly cancels the isovector part in the case of the first two components of both states in (20). This is a general property and follows from the fact that the flavour part in these two components corresponds to the mixed symmetric state $\phi_{\mathrm{M}, \mathrm{S}} \cdot{ }^{3}$ The proton

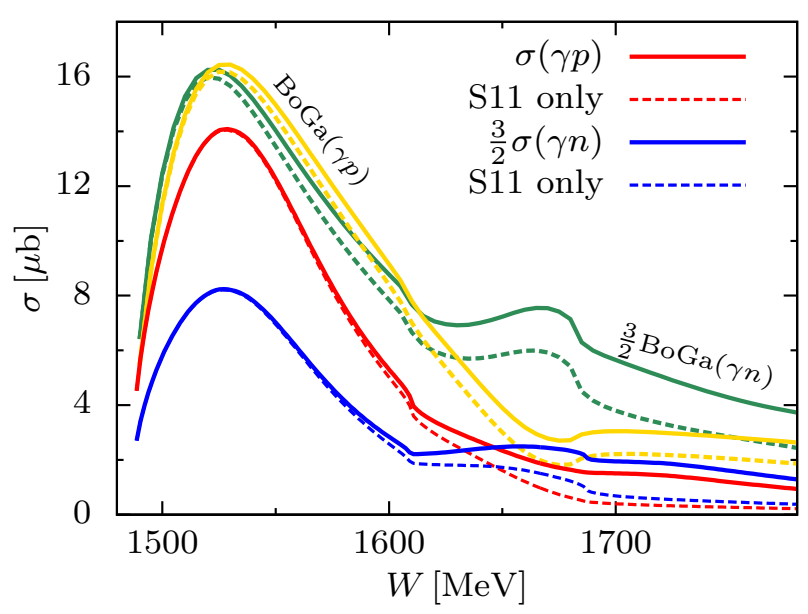

Fig. 6. (Color online.) The total cross-sections for $\gamma p \rightarrow \eta p$ and $\gamma n \rightarrow \eta n$ (multiplied by the conventional factor of $\frac{3}{2}$ ). Dashed lines: contribution of the $S_{11}$ partial wave. The BoGa curves have been reconstructed from the Bonn-Gatchina 20142 data set [68].

\footnotetext{
2 Readjusting the mixing angle could considerably improve the quality of the $\gamma n$ result but would seriously deteriorate the agreement for the other observables.

3 The property that the ${ }^{4} \mathbf{8}$ does not couple to the proton is known as the Moorhouse selection rule [64].
}

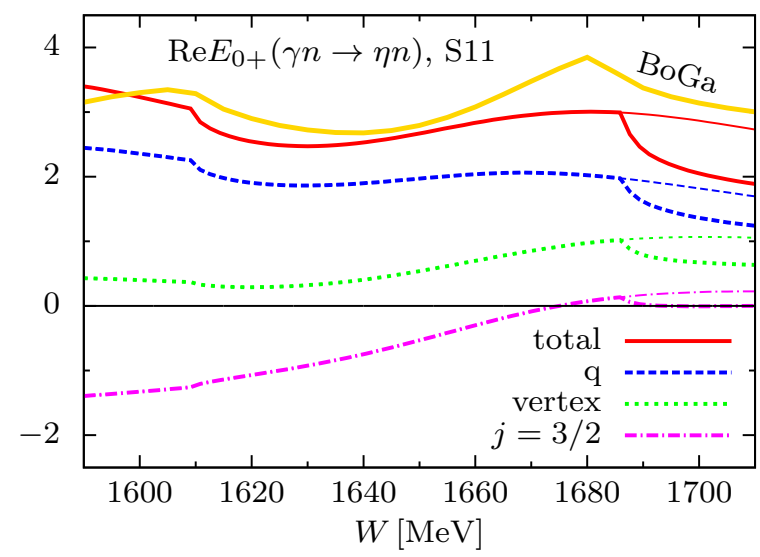

Fig. 7. (Color online.) Various contributions to the real part of the $E_{0+}$ amplitude: quark core (dashed), vertex correction (dotted), contribution from the $p_{3 / 2}$ orbit (dash-dotted) in units of $\mathrm{mfm}$. The Bonn-Gatchina results (thick yellow line at the top) is taken from the 2014-2 dataset and multiplied by -1 . The corresponding thin curves denote the solution without the $K \Sigma$ channel.

therefore receives no contribution from the $1 s \rightarrow 1 p_{3 / 2}$ transition. This is not the case with the neutron which receives contributions from all components in (20). The quark in the $1 p_{3 / 2}$ orbit has a distinctly different radial behaviour of the bispinor from that in the $1 p_{1 / 2}$ orbit (see Appendix C), which is reflected in a different momentumand $W$-behaviour of the amplitudes.

To illustrate this point we display in fig. 7 various contributions to the real part of the $E_{0+}$ photoproduction amplitude in the $\eta n$ channel in the region between the $K \Lambda$ and the $K \Sigma$ threshold. The contribution of the $p_{3 / 2}$ orbit is negative and is rising in this region, and influences the pure quark contribution and the contribution from the vertex correction in such a way that the total amplitude becomes flat or even slightly increases. When it reaches the $K \Sigma$ threshold it sharply drops as a consequence of the new channel opening (see fig. 2). Had there not been a new channel, a wide and shallow structure would have been observed. (We have chosen the case with the fixed mixing angle $-30^{\circ}$ since it sharpens the effect of the threshold compared to a smoother behaviour in fig. 6).

\section{$4.2 K^{+} \Lambda$ photoproduction}

At energies close to the threshold the $E_{0+}$ multipole represents the dominant contribution to $K$ photoproduction though not to the same extent as in the $\eta$ case (see fig. 8). Here our sign is again consistent with the Kaon MAID analysis [72] but opposite to that of the Bonn-Gatchina group. The strength of this multipole is, however, closer to the prediction of the Bonn-Gatchina group. For the $P_{13}$ wave our results agree with MAID, while it seems that we obtain a too strong contribution in the case of the $P_{11}$ wave. This contribution is sensitive to the admixture of the nucleon-pole term which remains important also at 

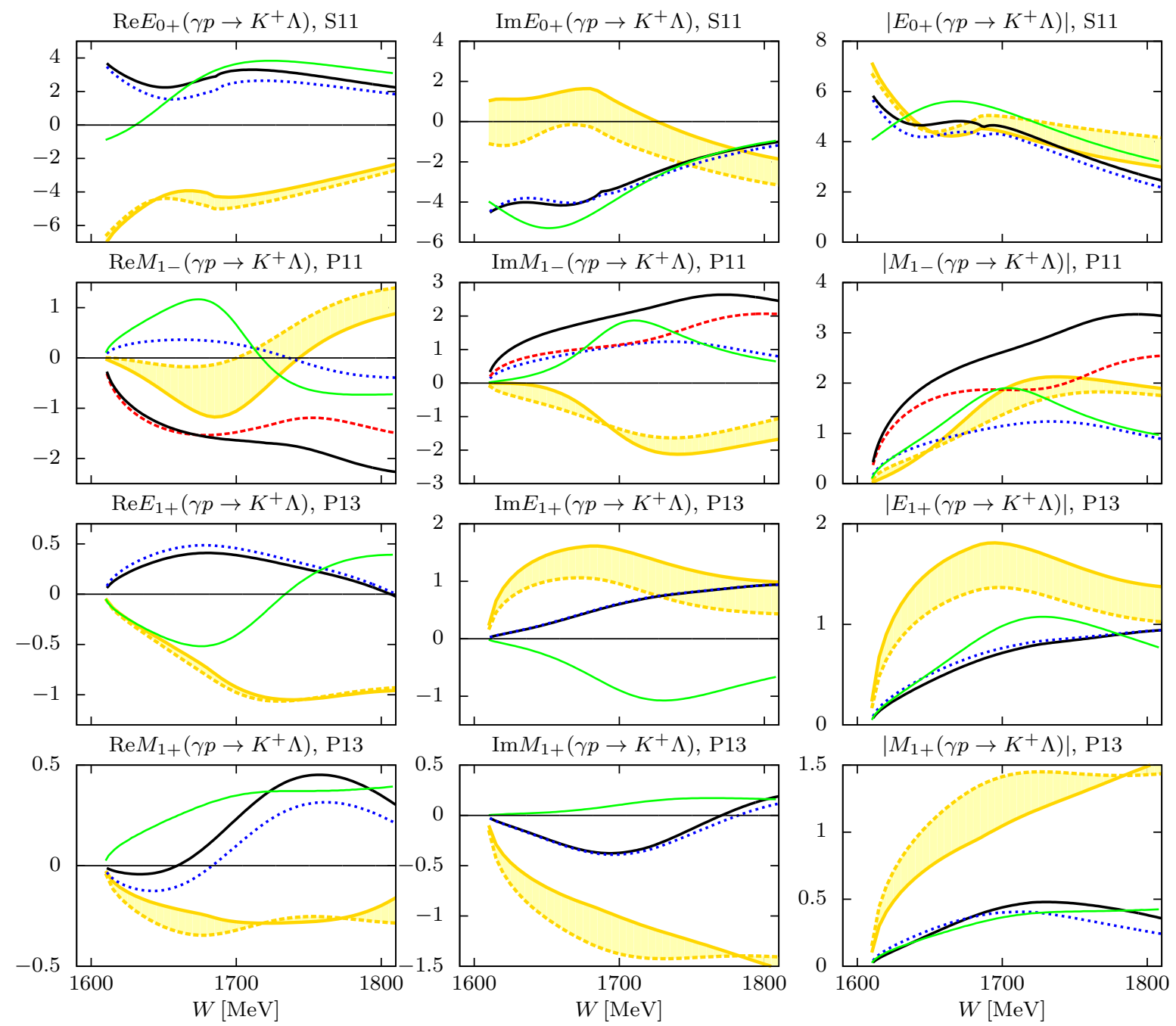

Fig. 8. (Color online.) The real and imaginary parts and the absolute values of multipoles dominating in $K^{+} \Lambda$ photoproduction. Notation as in fig. 5 .

higher energies. The trend can be seen in fig. 9 showing different contributions to the total cross-section. The Giessen analysis of this channel [41] also predicts the dominant contribution from the $S_{11}, P_{13}$ and $P_{11}$ waves with the $P_{13}$ being stronger than the $P_{11}$ one, opposite to our calculation but in agreement with the Bonn-Gatchina prediction. The total cross-section exhibits a surprisingly good agreement with the experimental points which, admittedly, could be a consequence of an overestimation of the strong part (see fig. 2) and an underestimation of the EM amplitudes.

In our model the main contribution to the background originates from the $t$-channel; at low energies the coupledchannel effect involving the $\gamma p \rightarrow \pi N$ process and the $T_{\pi N K \Lambda}$ matrix element turns out to be dominant but still weaker than the resonant one. We have not included the background involving the $K^{*}$ and $K_{1}$ mesons.

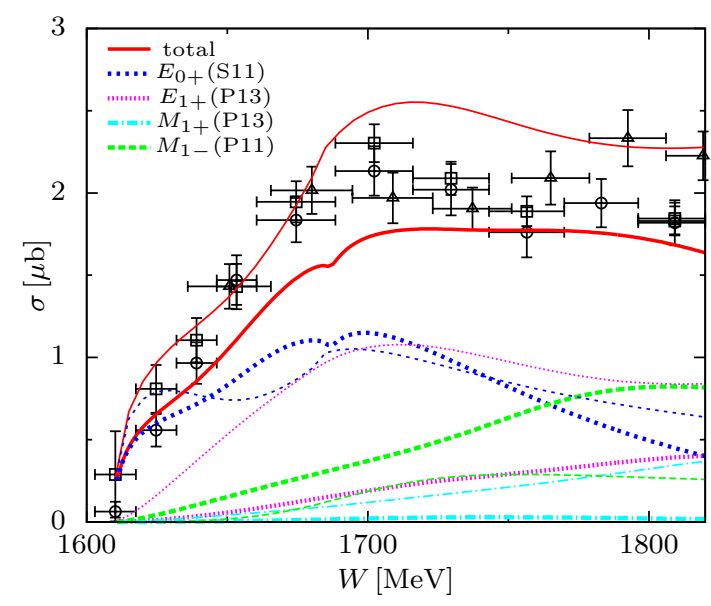

Fig. 9. (Color online.) Total cross-section for $\gamma p \rightarrow K^{+} \Lambda$. The experimental data are from [28-30]. The thin lines correspond to the cross-sections calculated from the Bonn-Gatchina 2014-1 dataset. 


\section{$4.3 K^{0} \Sigma$ photoproduction}

We have decided to concentrate only on $K^{0}$ photoproduction since the background processes in the case of $K^{+}$ photoproduction may considerably hinder the more interesting resonant contribution.

The amplitudes in this channel involve isospin-1/2 and isospin-3/2 contributions [55]:

$$
A\left(\gamma+p \rightarrow K^{0} \Sigma^{+}\right)=\sqrt{2}\left[A_{p}^{(1 / 2)}-\frac{1}{3} A^{(3 / 2)}\right], \quad A\left(\gamma+n \rightarrow K^{0} \Sigma^{0}\right)=-A_{n}^{(1 / 2)}+\frac{2}{3} A^{(3 / 2)} .
$$

In the case of the $E_{0+}$ multipole, the contribution from the $S_{31}$ partial wave turns out to be negligible in our model.
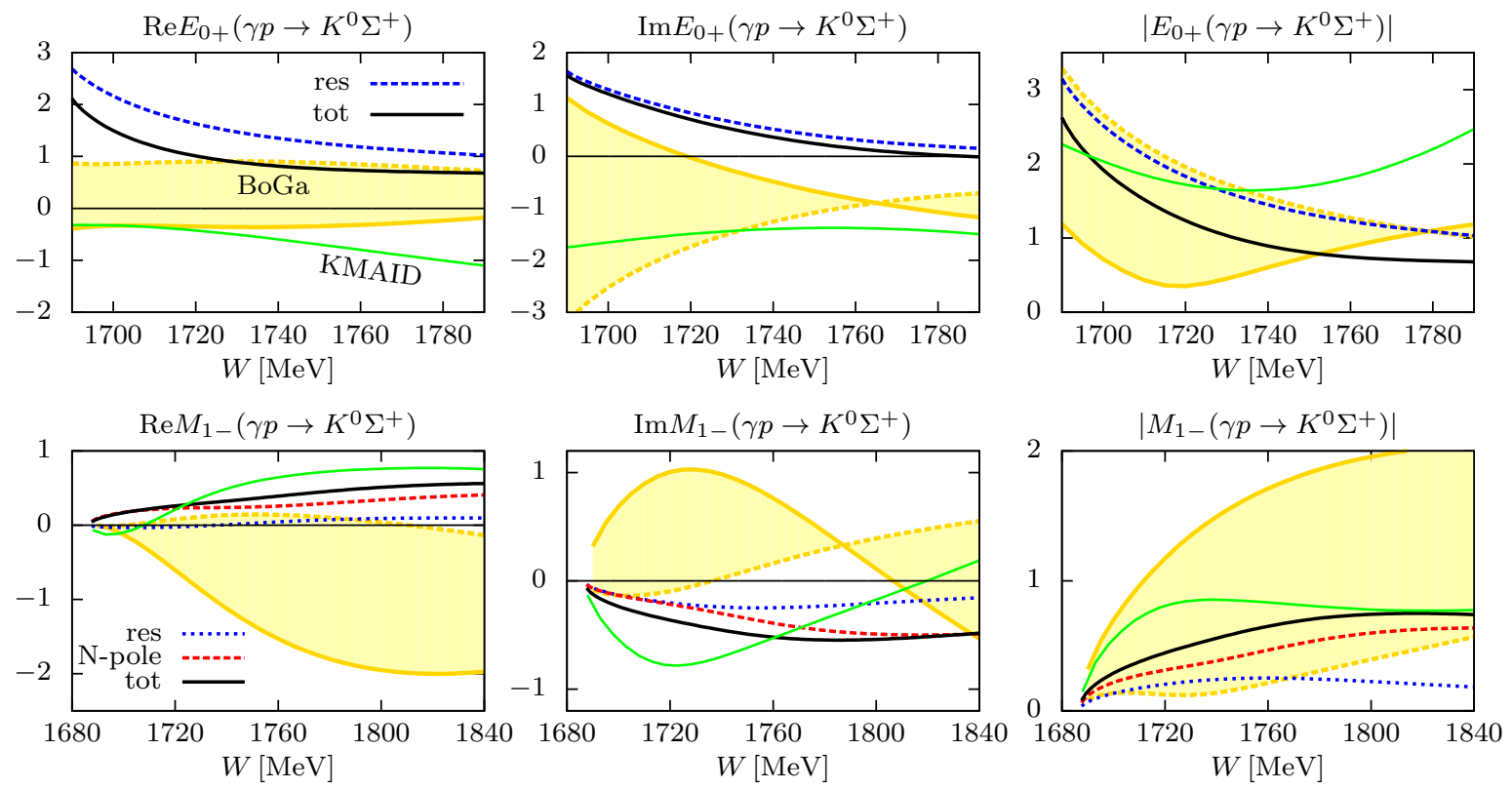

Fig. 10. (Color online.) The real and imaginary parts and the absolute values of the $E_{0+}$ and $M_{1-}$ photoproduction multipoles in the $K^{0} \Sigma^{+}$channel. Notation as in fig. 5 .
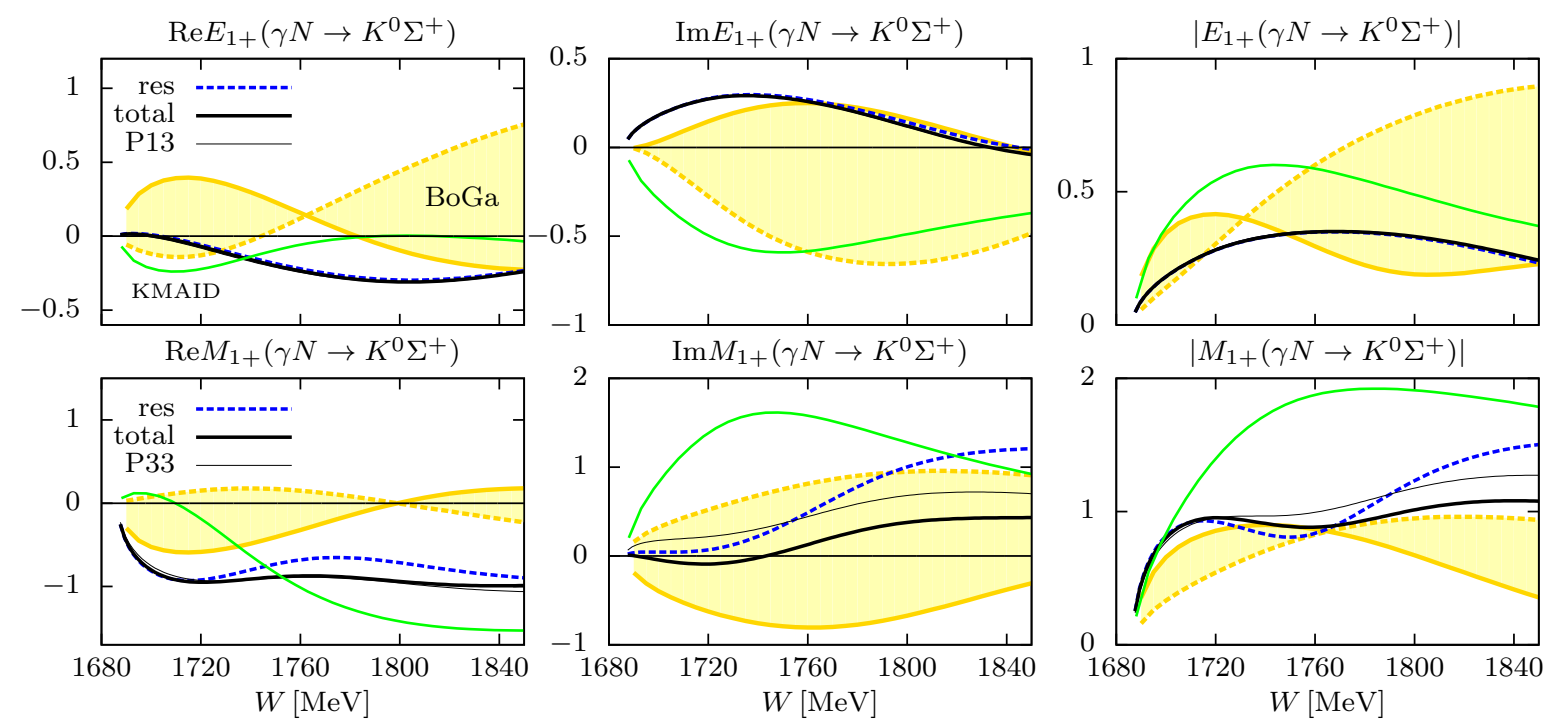

Fig. 11. (Color online.) The real and imaginary parts and the absolute values of the $E_{1+}$ and $M_{1+}$ photoproduction multipoles in the $K^{0} \Sigma^{+}$channel. Notation as in fig. 5. For the $E_{1+}\left(M_{1+}\right)$ multipole the leading $P_{13}\left(P_{33}\right)$ contribution is also shown (thin lines). 
In fig. 10 we display our results only for the isospin$1 / 2$ contribution and compare them to the relatively old Kaon MAID analysis [72] and to the recent, but still inconclusive, analysis of the Bonn-Gatchina group. The situation for the $M_{1-}$ multipole is similar; the contribution of the $P_{31}$ wave is negligible while the nucleon pole provides the dominant contribution. Let us notice that in our calculation only the $\Delta(1910) \frac{1}{2}^{+}$is assumed in this partial wave. In the case of the $M_{1+}$ and $E_{1-}$ amplitudes, both $P_{13}$ and $P_{33}$ partial waves contribute, however, in magnetic dipole transition the $P_{33}$ wave strongly dominates in analogy with pion photoproduction in the region of the $\Delta(1232)$ resonance. The situation is reversed for the $E_{1+}$ multipole; it is dominant in the $P_{13}$ wave since it involves an electric quadrupole transition of an $s$-wave quark to the $d$-state.

The role of the background terms in $K \Sigma$ is more important than in $K \Lambda$ production. They are dominated by the coupled-channel effects in which the amplitudes for the $t$-channel $\gamma p \rightarrow \pi N$ and $\gamma p \rightarrow \pi \Delta$ processes couple to the corresponding $T_{\pi N K \Sigma}$ and $T_{\pi \Delta K \Sigma}$ matrix elements. The background terms have the opposite sign with respect to the resonant ones and weaken most of the amplitudes in the $K^{0} \Sigma^{+}$channel with respect to those in the $K^{+} \Sigma^{0}$ channel, in agreement with the Giessen group analysis [20].

Different contributions to the total cross-section are displayed in fig. 12. Our results agree with the experimental values in the region close to the threshold and unambiguously favour the Bonn-Gatchina 2014-1 solution over the 2014-2 one. Not surprisingly, the $s$-wave kaons clearly dominate the threshold behaviour also in this channel. At higher energies, the $P_{33}$ and the $P_{13}$ waves become the leading terms followed by the $P_{11}$ wave, in agreement with the Bonn-Gatchina as well as the Giessen [20] predictions.

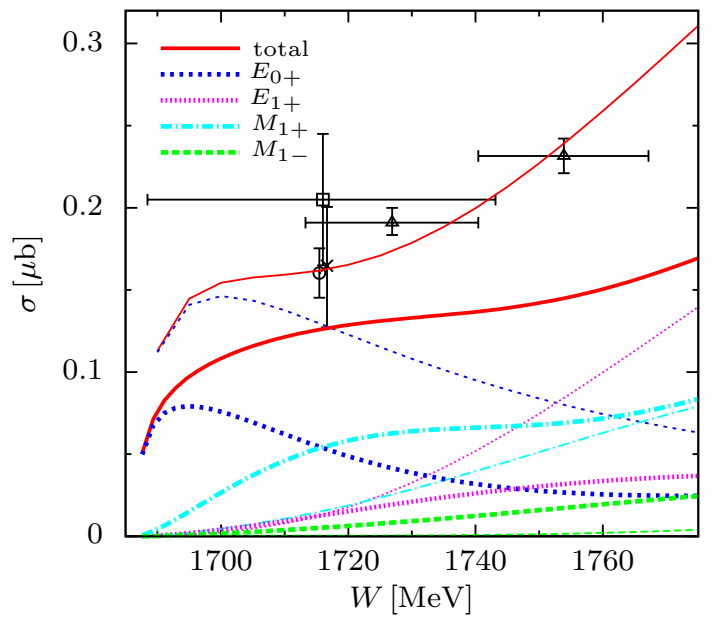

Fig. 12. (Color online.) Total cross-section for $\gamma p \rightarrow K^{0} \Sigma^{+}$ Experimental data are from [49,51-53]. Notation as in fig. 9.

\section{Conclusions}

We have proposed a relatively simple explanation for the resonance-like structure in the $\gamma n \rightarrow \eta n$ process at $1685 \mathrm{MeV}$ as a consequence of the property of the relativistic quark model which distinguishes between orbits with different $j$ on the one hand, and on the other hand as a coupledchannel effect due to the opening of the $K \Sigma$ channel. Note that the former effect could not be obtained in a non-relativistic constituent-quark model.

We have obtained a good overall agreement with observables involving $\eta N, K \Lambda$ and $K \Sigma$ channels by using a very small number of free parameters, which permits us to conclude that the low-lying resonances in the considered partial waves indeed fit very well in the quark-model picture. Furthermore, most excitations and decays can be well described in terms of single-quark transitions.

We have confirmed the leading role of the two lowest $S_{11}$ resonances in the threshold region found in several partial-wave analyses, as well as the relevance of the $P_{11}$, $P_{13}$ waves at somewhat higher energies for the isospin$1 / 2$ processes, and the $P_{33}$ wave for isospin- $3 / 2$ processes, while the $S_{31}$ and $D$-wave resonances play a minor role.

The SU(3) symmetry relations for the meson-baryon and photon-baryon coupling turn out to be well justified by the present calculation.

The resonance that may not fit in our scheme could be the $N(1710) \frac{1}{2}^{+}$. In our model the dominant contribution in the $P_{11}$ partial wave stems from the $N(1440) \frac{1}{2}^{+}$and the nucleon pole. If it turns out that the $N(1710) \frac{1}{2}^{+}$resonance indeed dominates the production of $\eta$ mesons and kaons, our assumption about the single-quark transition mechanism does not apply and one should seek an explanation beyond the simple quark model for the structure of this resonance.

\section{A The $P_{13}$ resonances}

The $L S$-coupled states belonging to the $\mathbf{7 0}$ multiplet recoupled to the $j j$-coupled basis read:

$$
\begin{aligned}
\left|\mathbf{7 0},{ }^{4} \mathbf{8}, J=\frac{3}{2}\right\rangle= & -\frac{2}{\sqrt{5}}\left|\mathbf{8}, \frac{3}{2}, S S A\right\rangle+\frac{1}{\sqrt{5}}\left|\mathbf{8}, \frac{3}{2}, S S B\right\rangle, \\
\left|\mathbf{7 0},{ }^{2} \mathbf{8}, J=\frac{3}{2}\right\rangle= & -\frac{1}{\sqrt{10}}\left|\mathbf{8}, \frac{3}{2}, S S A\right\rangle-\frac{1}{\sqrt{2}}\left|\mathbf{8}, \frac{3}{2}, S S A\right\rangle^{\prime} \\
& -\sqrt{\frac{2}{5}}\left|\mathbf{8}, \frac{3}{2}, S S B\right\rangle,
\end{aligned}
$$

where $S$ stands for the $s$-quark orbit and $A$ and $B$ for $j=\frac{3}{2}$ and $j=\frac{5}{2}$ orbits, respectively. The state with total $J=\frac{3}{2}$ and $M_{J}=\frac{3}{2}$ is constructed as

$$
\begin{aligned}
\left|\boldsymbol{8}, \frac{3}{2}, S S X\right\rangle= & \frac{1}{\sqrt{2}}\left[\phi_{11}^{[21]}\left|\frac{3}{2} \frac{3}{2}, S S X\right\rangle_{11}^{[21]}\right. \\
& \left.+\phi_{21}^{[21]}\left|\frac{3}{2} \frac{3}{2}, S S X\right\rangle_{21}^{[21]}\right]
\end{aligned}
$$


where

$$
\begin{aligned}
\left|\frac{3}{2} \frac{3}{2}, S S A\right\rangle_{11}^{[21]}= & \frac{1}{\sqrt{60}}[2(2 \uparrow \uparrow \beta-\uparrow \beta \uparrow-\beta \uparrow \uparrow) \\
& -\sqrt{3}(2 \uparrow \downarrow \alpha+2 \downarrow \uparrow \alpha \\
& -\uparrow \alpha \downarrow-\alpha \uparrow \downarrow-\downarrow \alpha \uparrow-\alpha \downarrow \uparrow)], \\
\left|\frac{3}{2} \frac{3}{2}, S S A\right\rangle_{21}^{[21]}= & \frac{1}{\sqrt{20}}[2(\uparrow \beta \uparrow-\beta \uparrow \uparrow) \\
& -\sqrt{3}(\uparrow \alpha \downarrow-\alpha \uparrow \downarrow+\downarrow \alpha \uparrow-\alpha \downarrow \uparrow)], \\
\left|\frac{3}{2} \frac{3}{2}, S S A\right\rangle_{12}^{[21]^{\prime}=} & \frac{1}{2}[\uparrow \alpha \downarrow+\alpha \uparrow \downarrow-\downarrow \alpha \uparrow-\alpha \downarrow \uparrow], \\
\left|\frac{3}{2} \frac{3}{2}, S S A\right\rangle_{22}^{[21]^{\prime}=} & \frac{1}{\sqrt{12}}[2 \uparrow \downarrow \alpha-2 \downarrow \uparrow \alpha \\
& +\uparrow \alpha \downarrow-\alpha \uparrow \downarrow+\alpha \downarrow \uparrow-\downarrow \alpha \uparrow], \\
\left|\frac{3}{2} \frac{3}{2}, S S B\right\rangle_{11}^{[21]}= & \frac{1}{\sqrt{90}}[\sqrt{10}(2 \downarrow \downarrow a-\downarrow a \downarrow-a \downarrow \downarrow) \\
& -\sqrt{2}(2 \uparrow \downarrow b+2 \downarrow \uparrow b \\
& -\uparrow b \downarrow-b \uparrow \downarrow-\downarrow b \uparrow-b \downarrow \uparrow) \\
& +(2 \uparrow \uparrow c-\uparrow c \uparrow-c \uparrow \uparrow)], \\
\left|\frac{3}{2} \frac{3}{2}, S S B\right\rangle_{21}^{[21]}= & \frac{1}{\sqrt{30}}[\sqrt{10}(\downarrow a \downarrow-a \downarrow \downarrow) \\
& -\sqrt{2}(\uparrow b \downarrow+b \uparrow \downarrow-\downarrow b \uparrow-b \downarrow \uparrow) \\
& +(\uparrow c \uparrow-c \uparrow \uparrow)],
\end{aligned}
$$

and $\uparrow=\left(j=\frac{1}{2}, m_{j}=\frac{1}{2}\right), \downarrow=\left(\frac{1}{2},-\frac{1}{2}\right)$;

$\alpha=\left(j=\frac{3}{2}, m_{j}=\frac{3}{2}\right), \beta=\left(\frac{3}{2}, \frac{1}{2}\right)$;

$a=\left(j=\frac{5}{2}, m_{j}=\frac{5}{2}\right), b=\left(\frac{5}{2}, \frac{3}{2}\right), c=\left(\frac{5}{2}, \frac{1}{2}\right)$.

Here $\phi_{s t}^{[21]}$ are the flavour wave functions: $\phi_{11}^{[21]}=$ $\sqrt{1 / 6}(2 u u d-u d u-d u u), \phi_{21}^{[21]}=\sqrt{1 / 2}(u d u-d u u)$.

\section{B The meson-quark vertices}

The meson-quark vertices $V^{M}(k)$ in (1) are evaluated in the Cloudy Bag Model assuming that one of the three quarks is excited from the $1 s$ orbit to an $l$-wave orbit with $j=l \pm \frac{1}{2}$.

For the pion with orbital momentum $L$, its third component $M$ and the third component of isospin $t$ we have

$$
\begin{aligned}
V_{L M t}^{\pi}(k) & =\frac{\sqrt{3}}{2 \pi f} \sum_{l, j=l \pm \frac{1}{2}} \sqrt{2 l+1} C_{10 l 0}^{L 0} W\left(L l \frac{1}{2} \frac{1}{2} ; 1 j\right) \\
& \times(-1)^{l+\frac{1}{2}-j} \sqrt{\frac{\omega_{s} \omega_{l j}}{\left(\omega_{s}-1\right)\left(\omega_{l j} \mp\left(j+\frac{1}{2}\right)\right)}} \\
& \times \frac{k^{2}}{\sqrt{2 \omega_{k}}} \frac{j_{L}\left(k R_{\mathrm{bag}}\right)}{k R_{\mathrm{bag}}} \sum_{i=1}^{3} \Sigma_{L M}^{\left[j \frac{1}{2}\right]}(i) \tau_{t}(i),
\end{aligned}
$$

where

$$
\left\langle j m_{j}\left|\Sigma_{L M}^{\left[j \frac{1}{2}\right]}\right| \frac{1}{2} m_{s}\right\rangle=C_{\frac{1}{2} m_{s} L M}^{j m_{j}}, \quad \Sigma_{1 M}^{\left[\frac{1}{2} \frac{1}{2}\right]}=\frac{1}{\sqrt{3}} \sigma_{M},
$$

and $\omega_{l j}$ is a solution of the boundary condition equation for massless quarks: $j_{l}\left(\omega_{l j} R_{\mathrm{bag}}\right)= \pm j_{l \pm 1}\left(\omega_{l j} R_{\mathrm{bag}}\right), j=$ $l \pm \frac{1}{2}$; where $j_{l}$ are the spherical Bessel functions, and $W(\ldots)$ are the Racah coefficients.

For the $\eta$ meson the $\tau_{t}(i)$ operator is replaced by the $\lambda_{8}(i)$ operator, and for the kaons by the $V_{t}(i)+U_{t}(i)$ operator.

In our approach the state of the $\rho$ meson is specified by its total angular momentum $J_{\rho}$ and its third component $M$, orbital angular momentum $L$, and isospin $t$. It is related to the state in which the total spin $S$ of the $\rho N$ system is specified:

$\left|S L J M_{J}\right\rangle=\sum_{J_{\rho}} \sqrt{2 S+1} \sqrt{2 J_{\rho}+1} W\left(L J 1 \frac{1}{2} ; S J_{\rho}\right)\left|J_{\rho} L J M_{J}\right\rangle$,

where $J$ and $M_{J}$ refer to the total angular momentum of the $\rho N$ system. The corresponding vertex operator is given by

$$
\begin{aligned}
V_{J_{\rho} L M t}^{\rho}(k) & =\frac{3}{2 \pi f_{\rho}}(-1)^{J_{\rho}+L} \sum_{l, j=l \pm \frac{1}{2}} \sqrt{2 J_{\rho}+1} \sqrt{2 l+1} C_{10 l 0}^{L 0} \\
& \times W\left(l L 11 ; 1 J_{\rho}\right) W\left(J_{\rho} l \frac{1}{2} \frac{1}{2} ; 1 j\right) \frac{k^{2}}{\sqrt{2 \omega_{k}}} \frac{j_{L}\left(k R_{\mathrm{bag}}\right)}{k R_{\mathrm{bag}}} \\
& \times \sqrt{\frac{\omega_{s} \omega_{l j}}{\left(\omega_{s}-1\right)\left(\omega_{l j} \mp\left(j+\frac{1}{2}\right)\right)}} \sum_{i=1}^{3} \Sigma_{J_{\rho} M}^{\left[j \frac{1}{2}\right]}(i) \tau_{t}(i) .
\end{aligned}
$$

\section{The $E$ and $M$ multipole operators}

We give here the expressions for the quark part of the transverse magnetic and electric multipole operators involving transition from an $s$-wave quark orbit to an $l$-wave quark orbit with $j=l \pm \frac{1}{2} ; u_{s}, v_{s}$ and $u_{l j}, v_{l j}$ are the corresponding bispinors.

$$
\begin{aligned}
& \int \mathrm{d} \boldsymbol{r} j^{q} \cdot \boldsymbol{A}_{L M}^{m}=\mathrm{i}^{L+1} \sqrt{2 L+1} \int \mathrm{d} r r^{2} j_{L}(q r) \\
& \times \sum_{l, j=l \pm \frac{1}{2}}\left\{(-1)^{L+j-\frac{1}{2}} C_{10 L 0}^{L 0} \delta_{l, L}\left(u_{l j} v_{s}-v_{l j} u_{s}\right)\right. \\
&+6 \sqrt{2 L+1} \sqrt{2 l+1} W(l L 11 ; 1 L) W\left(L l_{\left.\frac{1}{2} \frac{1}{2} ; 1 j\right)}\right. \\
&\left.\times C_{10 l 0}^{L 0}\left(u_{l j} v_{s}+v_{l j} u_{s}\right)\right\}\left[\frac{1}{6}+\frac{1}{2} \tau_{0}(i)\right] \Sigma_{L M}^{\left[j \frac{1}{2}\right]}, \\
& \int \mathrm{d} \boldsymbol{r} \boldsymbol{j}^{q} \cdot \boldsymbol{A}_{L 1}^{e}=\mathrm{i}^{L} \int \mathrm{d} r r^{2} \sum_{l, j=l \pm \frac{1}{2}}\left\{\sqrt{L+1} j_{L-1}(q r)\right. \\
& \times\left[(-1)^{L+j+\frac{1}{2}} C_{10 L 0}^{L-10} \delta_{l, L}\left(u_{l j} v_{s}-v_{l j} u_{s}\right)\right. \\
& \quad-6 \sqrt{2 L+1} \sqrt{2 l+1} W(l L-111 ; 1 L) W\left(L l_{\frac{1}{2} \frac{1}{2}} ; 1 j\right) \\
& \quad \times C_{10 l 0}^{\left.L-10\left(u_{l j} v_{s}+v_{l j} u_{s}\right)\right]}
\end{aligned}
$$




$$
\begin{aligned}
& -\sqrt{L} j_{L+1}(q r)\left[(-1)^{L+j+\frac{1}{2}} C_{10 L 0}^{L+10} \delta_{l, L}\left(u_{l j} v_{s}-v_{l j} u_{s}\right)\right. \\
& -6 \sqrt{2 L+1} \sqrt{2 l+1} W(l L+111 ; 1 L) W\left(L l_{\frac{1}{2} \frac{1}{2}} ; 1 j\right) \\
& \left.\left.\times C_{10 l 0}^{L+10}\left(u_{l j} v_{s}+v_{l j} u_{s}\right)\right]\right\}\left[\frac{1}{6}+\frac{1}{2} \tau_{0}(i)\right] \Sigma_{L 1}^{\left[j \frac{1}{2}\right]},
\end{aligned}
$$

where $q=|\boldsymbol{q}|$ is the photon momentum.

For the photon-pion interaction the corresponding operators are written in the form

$$
\begin{aligned}
& \int \mathrm{d} \boldsymbol{r} \boldsymbol{j}_{\pi} \cdot \boldsymbol{A}_{L M}^{m}=-\mathrm{i}^{L+1} \sqrt{2} \sum_{l l^{\prime}}(-1)^{L-1+l-l^{\prime}} \sqrt{2 l+1} \\
& \times \frac{2}{\pi} \int \mathrm{d} r r^{2} \int \frac{\mathrm{d} k k}{\sqrt{2 \omega_{k}}} \int \frac{\mathrm{d} k^{\prime} k^{\prime 2}}{\sqrt{2 \omega_{k}^{\prime}}} j_{l}(k r) j_{L}(q r) \\
& \times\left[j_{l^{\prime}+1}\left(k^{\prime} r\right) \sqrt{\left(l^{\prime}+1\right)\left(2 l^{\prime}+3\right)} C_{l 0 l^{\prime}+10}^{L 0} W\left(l^{\prime} 1 l L ; l^{\prime}+1, L\right)\right. \\
& \left.+j_{l^{\prime}-1}\left(k^{\prime} r\right) \sqrt{l^{\prime}\left(2 l^{\prime}-1\right)} C_{l 0 l^{\prime}-10}^{L 0} W\left(l^{\prime} 1 l L ; l^{\prime}-1, L\right)\right] \\
& \times \mathbf{A}_{l l^{\prime} L M}^{10}, \\
& \int \mathrm{d} \boldsymbol{r} \boldsymbol{j}_{\pi} \cdot \boldsymbol{A}_{L 1}^{e}=-\mathrm{i}^{L+2} \sum_{l l^{\prime}}(-1)^{L-1+l-l^{\prime}} \frac{\sqrt{2} \sqrt{2 l+1}}{\sqrt{L(L+1)}} \\
& \times \frac{2}{\pi} \int \mathrm{d} r r^{2} \int \frac{\mathrm{d} k k}{\sqrt{2 \omega_{k}}} \int \frac{\mathrm{d} k^{\prime}{k^{\prime}}^{2}}{\sqrt{2 \omega_{k}^{\prime}}} j_{l}(k r) \\
& \times\left\{-L \sqrt{L+1} j_{L+1}(q r)\right. \\
& \times\left[j_{l^{\prime}+1}\left(k^{\prime} r\right) \sqrt{\left(l^{\prime}+1\right)\left(2 l^{\prime}+3\right)} C_{l 0 l^{\prime}+10}^{L+10}\right. \\
& \times W\left(l^{\prime} 1 l L+1 ; l^{\prime}+1, L\right) \\
& +j_{l^{\prime}-1}\left(k^{\prime} r\right) \sqrt{l^{\prime}\left(2 l^{\prime}-1\right)} C_{l 0 l^{\prime}-10}^{L+10} \\
& \left.\times W\left(l^{\prime} 1 l L+1 ; l^{\prime}-1, L\right)\right] \\
& +(L+1) \sqrt{L} j_{L-1}(q r) \\
& \times\left[j_{l^{\prime}+1}\left(k^{\prime} r\right) \sqrt{\left(l^{\prime}+1\right)\left(2 l^{\prime}+3\right)} C_{l 0 l^{\prime}+10}^{L-10}\right. \\
& \times W\left(l^{\prime} 1 l L-1 ; l^{\prime}+1, L\right) \\
& +j_{l^{\prime}-1}\left(k^{\prime} r\right) \sqrt{l^{\prime}\left(2 l^{\prime}-1\right)} C_{l 0 l^{\prime}-10}^{L-10} \\
& \left.\left.\times W\left(l^{\prime} 1 l L-1 ; l^{\prime}-1, L\right)\right]\right\} \\
& \times \mathbf{A}_{l l^{\prime} L 1}^{10}
\end{aligned}
$$

Here $\mathbf{A}_{l l^{\prime} 10}^{L 1}$ is the product of the $l$ - and $l^{\prime}$-wave pion creation and annihilation operators coupled to angular momentum $L$ and isospin 1 . Note that the orbital angular momentum of the pion can be changed. For the kaons, the expressions differ only in the coefficients of the isospin coupling.

\section{References}

1. P. Alberto, L. Amoreira, M. Fiolhais, B. Golli, and S. Širca, Eur. Phys. J. A 26, 99 (2005).
2. B. Golli and S. Širca, Eur. Phys. J. A 38, 271 (2008).

3. B. Golli, S. Širca, and M. Fiolhais, Eur. Phys. J. A 42, 185 (2009).

4. B. Golli, S. Širca, Eur. Phys. J. A 47, 61 (2011).

5. B. Golli, S. Širca, Eur. Phys. J. A 49, 111 (2013).

6. V. Credé et al. (CBELSA Collaboration), Phys. Rev. Lett. 94, 012004 (2005).

7. O. Bartalini et al. (GRAAL Collaboration), Eur. Phys. J. A 33, 169 (2007).

8. V. Credé et al. (CBELSA/TAPS Collaboration), Phys. Rev. C 80, 055202 (2009).

9. M. Williams et al. (CLAS Collaboration), Phys. Rev. C 80, 045213 (2009).

10. E. F. McNicoll et al. (Crystal Ball Collaboration), Phys. Rev. C 82, 035208 (2010).

11. D. Werthmüller et al. (A2 Collaboration), Phys. Rev. C 90, 015205 (2014).

12. D. Rebreyend et al. (GRAAL Collaboration), Int. J. Mod. Phys. A 20, 1554 (2005).

13. I. Jaegle et al. (CBELSA/TAPS Collaboration), Phys. Rev. Lett. 100, 252002 (2008).

14. I. Jaegle et al. (CBELSA/TAPS Collaboration), Eur. Phys. J. A 47, 89 (2011).

15. V. Kuznetsov et al., Phys. Lett. B 647, 23 (2007).

16. Ya. Azimov et al. Eur. Phys. J. A 25, 325 (2005).

17. M. Döring, K. Nakayama, Phys. Lett. B 683, 145 (2010).

18. R. Shyam and O. Scholten, Phys. Rev. C 78, 065201 (2008).

19. V. Shklyar, H. Lenske, U. Mosel, Phys. Lett. B 650, 172 (2007).

20. V. Shklyar, H. Lenske, U. Mosel, Phys. Rev. C 87, 015201 (2013).

21. A. V. Anisovich et al., Eur. Phys. J. A 41, 13 (2009).

22. A. V. Anisovich et al., Eur. Phys. J. A 51, 72 (2015).

23. X.-H. Zhong, Q. Zhao, Phys. Rev. C 84, 045207 (2011).

24. Jun He, B. Saghai, Phys. Rev. C 80, 015207 (2009).

25. Jun He, B. Saghai, Phys. Rev. C 82, 035206 (2010).

26. D. Ruić, M. Mai, U.-G. Meißner, Phys. Lett. B 704659 (2011).

27. M. Mai, P. C. Bruns, and U.-G. Meißner, Phys. Rev. D 86, 094033 (2012).

28. M. Bockhorst et al. (SAPHIR Collaboration), Z. Phys. C 63, 37 (1994).

29. M. Q. Tran et al. (SAPHIR Collaboration), Phys. Lett. B 445, 20 (1998).

30. K. H. Glander et al. (SAPHIR Collaboration), Eur. Phys. J. A 19, 251 (2004).

31. R. Bradford et al. (CLAS Collaboration), Phys. Rev. C 73, 035202 (2006).

32. J. W. C. McNabb et al. (CLAS Collaboration), Phys. Rev. C 69, 042201(R) (2004).

33. M. Sumihama et al. (LEPS Collaboration), Phys. Rev. C 73, 035214 (2006).

34. A. Lleres et al. (GRAAL Collaboration), Eur. Phys. J. A 31, 79 (2007).

35. A. Lleres et al. (GRAAL Collaboration), Eur. Phys. J. A 39, 149 (2009).

36. M. E. McCracken et al. (CLAS Collaboration), Phys. Rev. C 81, 025201 (2010).

37. Z. Li, Rev. C 52, 1648 (1995).

38. Z. Li, M. Wei-Hsing, Z. Lin, Rev. C 54, R2171 (1996).

39. S. Steininger and Ulf-G. Meißner, Phys. Lett. B 391, 446 (1997). 
40. B. Borasoy, P. C. Bruns, U.-G. Meissner, and R. Nissler, Eur. Phys. J. A 34, 161 (2007).

41. V. Shklyar, H. Lenske, U. Mosel, Phys. Rev. C 72, 015210 (2005).

42. R. Shyam, O. Scholten, and H. Lenske, Phys. Rev. C 81, 015201 (2010).

43. L. De Cruz, J. Ryckebusch, T. Vrancx, and P. Vancraeyveld, Phys. Rev. C 86, 015212 (2012).

44. T. Mart, Phys. Rev. C 82, 025209 (2010).

45. T. Mart, Phys. Rev. C 83, 048203 (2011).

46. M. Shrestha and D. M. Manley, Phys. Rev. C 86, 045204 (2012).

47. M. Shrestha and D. M. Manley, Phys. Rev. C 86, 055203 (2012).

48. S. Goers et al. (SAPHIR Collaboration), Phys. Lett. B 464, 331 (1999).

49. R. Lawall et al. (SAPHIR Collaboration), Eur. Phys. J. A 24, 275 (2005).

50. F. J. Klein et al. (CLAS Collaboration), Nucl. Phys. A 754, 321(2005).

51. R. Castelijns et al. (CBELSA/TAPS Collaboration), Eur. Phys. J. A 35, 39 (2008).

52. R. Ewald et al. (CBELSA/TAPS Collaboration), Phys. Lett. B 713, 180 (2012).

53. P. Aguar-Bartolomé et al. (A2 Collaboration), Phys. Rev. C 88, 044601 (2013).

54. A. V. Anisovich et al., Eur. Phys. J. A 49, 158 (2013).

55. T. Mart, Phys. Rev. C 90, 065202 (2014).

56. D. Rönchen et al., Eur. Phys. J. A 49, 44 (2013).

57. H. Kamano, S. X. Nakamura, T.-S. H. Lee, T. Sato, Phys. Rev. C 88, 035209 (2013).

58. E. A. Veit, B. K. Jennings, A. W. Thomas, R. C. Barret, Phys. Rev. D 31, 1033 (1985).

59. Gerald A. Miller, and Anthony W. Thomas, Phys. Rev. C 56, 2329 (1997).

60. D. H. Lu, A. W. Thomas, and A. G. Williams, Phys. Rev. C 55, 3108 (1997).

61. N. Isgur and G. Karl. Phys. Lett. 72B, 109 (1977).

62. T. A. deGrand, Ann. Phys. 101, 496 (1976).

63. F. Myhrer and J. Wroldsen, Z. Phys. C 25, 281 (1984).

64. R. G. Moorhouse, Phys. Rev. Lett. 16772 (1996).

65. R. A. Arndt, W. J. Briscoe, I. I. Strakovsky, and R. L. Workman, Phys. Rev. C 74, 045205 (2006).

66. http://gwdac.phys.gwu.edu/analysis/pr_analysis.html.

67. P. Alberto, M. Fiolhais, B. Golli, and J. Marques, Phys. Lett. B 523, 273 (2001).

68. http://pwa.hiskp.uni-bonn.de/.

69. M. Fiolhais, B. Golli, S. Širca, Phys. Lett. B 373, 229 (1996).

70. D. M. Manley, E. M. Saleski, Phys. Rev. D 45, 4002 (1992).

71. L. Tiator, $\eta$ MAID 2015, private communication.

72. F. X. Lee, T. Mart, C. Bennhold, H. Haberzettl, L. E. Wright, Nucl. Phys. A 695, 237 (2001); see also http://portal.kph. uni-mainz.de/MAID/kaon/. 